\title{
Mobile KIS-Systeme
}

Den drahtlosen Zugang zum Krankenhausinformationssystem (KIS) am Patientenbett gibt es schon länger. Nun gehen einige Hersteller noch einen Schritt weiter und liefern Softwarelösungen, die den Zugriff auf das KIS auch mit handelsüblichen Smartphones und Tablets ermöglichen. Sie lassen sich an jedem Ort einer Klinik nutzen.

Mobile Geräte sind heute ständige Begleiter. Nicht nur Jugendliche legen ihre Smartphones kaum mehr aus der Hand auch die Erwachsenen benutzen sie mehrmals täglich. Laut einer Onlinestudie von ARD und ZDF aus dem Jahr 2014 werden Smartphones und Tablets mittlerweile stärker genutzt als herkömmliche Handys. Ganze 98\% des MobiltelefonUmsatzes entfallen laut dem IT-Verband Bitkom auf Smartphones. Inzwischen verwenden 63\% der Bevölkerung ab 14 Jahren ein Smartphone oder Tablet 2013 waren es erst 41\%. Da verwundert es nicht, dass auch das Klinikpersonal die Vorteile der modernen Kommunikation, die in der Industrie und dem Handel längst zum Standard gehört, voll ausschöpfen will.

\section{Mobil ins KIS}

Trotzdem hinkt das Gesundheitswesen dieser Entwicklung etwas hinterher. Zwar nutzt man auch hier immer öfter Geräte wie mobile Visitewägen und Laptops. Allerdings müssen sich Ärzte und Pfleger meist dennoch an den PC-Arbeitsplatz setzen, wenn sie Patientendaten abrufen, Fälle dokumentieren oder Behandlungsanweisungen geben wollen. Das hat auch die Industrie erkannt. Einige Hersteller bieten mittlerweile Softwarelö- sungen, die den Zugriff auf das KIS auch per Mobilgerät erlauben - und damit den Zugang zu sämtlichen Behandlungs- und Untersuchungsdaten, die in einem Krankenhaus vorliegen. Die Softwarelösung gibt es in Form einer Applikation (APP), die sich auf herkömmlichen Smartphones und Tablets installieren lässt. Anschließend ermöglicht sie den mobilen Zugriff auf das KIS über WLAN, das in vielen Kliniken flächendeckend verfügbar ist. Um die KIS-Daten zu schützen, ist das WLAN verschlüsselt. Zusätzlich müssen die Nutzer der APPs ihr Gerät vorher im KIS authentifizieren. Die Geräte sind dabei reine Ein- und Ausgabemedien, die Daten selbst bleiben stets im hausinternen Rechenzentrum. Alle Geräte werden zentral ver-

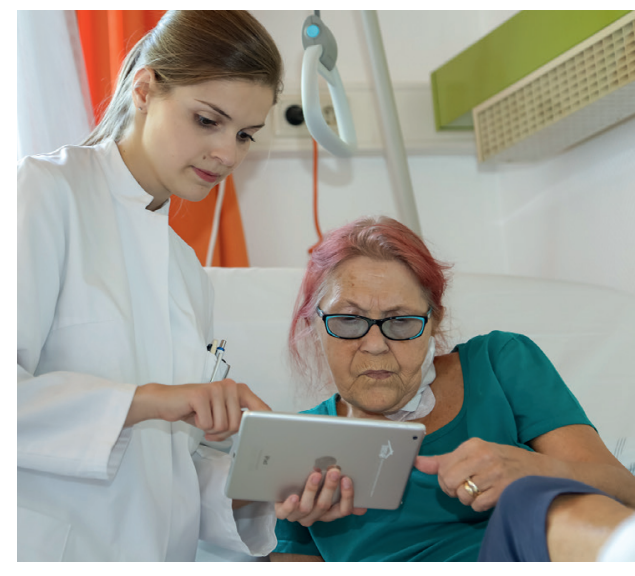

Abb.1 Umfassend informiert: Mit den neuen KIS-APPs kann das Klinikpersonal Patienten stets über aktuelle Behandlungsschritte oder Untersuchungsergebnisse informieren, ohne dafür auf Visitewägen oder Patienten-Entertainmentsysteme zurückgreifen zu müssen.

waltet, sodass Zugriffsrechte jederzeit gesperrt oder die APP auf einzelnen Geräten von außen gelöscht werden kann.

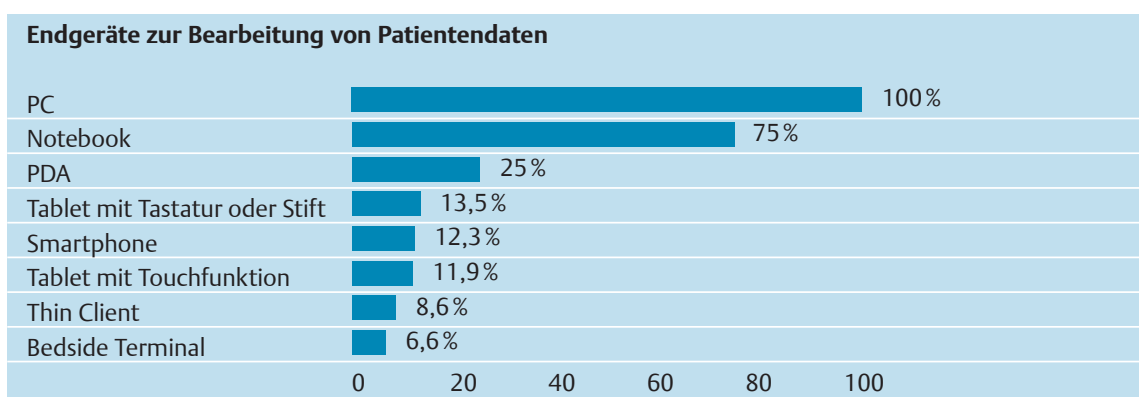

Abb. 1 Zukunftstrend: Das Klinikpersonal muss sich meist an den PC setzen, um Patientendaten zu bearbeiten. Allerdings nutzen bereits $12 \%$ der deutschen Krankenhäuser dafür ein Smartphone. Quelle: IT Report Gesundheitswesen 2014 


\section{Zeitgewinn durch Tablet-PCs}

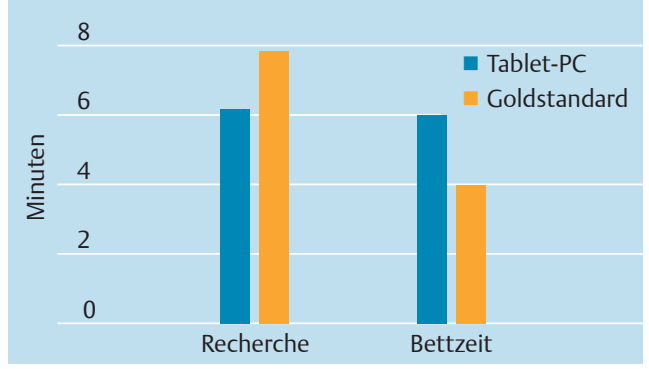

Abb. 2 Patientenfreundlich: Auch die Recherchezeit kann der Einsatz eines Tablet-PCs oder anderer mobiler Endgeräte verkürzen. Das lässt mehr Raum für den Patientenkontakt.

Quelle: Studie zur mobilen Visite 2014, Telekom Healthcare

\section{Auch außerhalb der Klinik nutzbar}

Eine solche APP hat das ehemals zum finnischen IT-Unternehmen Tieto gehörende Team gemeinsam mit dem Knappschaftskrankenhaus Bottrop bereits seit 2005 entwickelt. Daraus ist die App „Imed One Mobile” entstanden, die anschließend von Telekom Healthcare speziell für ihr hauseigenes KIS-System weiterentwickelt wurde. „Imed One Mobile” ist inzwischen weltweit in rund 40 Kliniken im Einsatz. Damit können die Ärzte und Pflegekräfte zum Beispiel über ein werte, Termine und Aufträge in das KIS eingeben. Auch klinische Daten wie Röntgenbilder und Laborwerte lassen sich vor Ort abrufen. Mit den mobilen Endgeräten können Anwender sogar Zusatzfunktionen wie die eingebaute Kamera nutzen. So kann das Personal Wunden fotografieren, den Wundverlauf erfassen und in der digitalen Akte speichern. „Der Vorteil ist, dass sie diese Bilder im Gegensatz zu Digitalkameras direkt mit der Patientenakte verknüpfen: Man macht das Bild, und sofort verknüpft die Software es mit dem Dokument. Gleichzeitig wird eingestanzt, $\mathrm{zu}$ welchem Bild welcher Patient gehört - und wann die Aufnahme gemacht wurde", erklärt Jan Ehlting, der Erfinder von iPad mini direkt am Krankenbett Vital-

Imed One Mobile und Leiter für mobile Gesundheitslösungen bei Telekom Healthcare. Führungskräfte können sich damit sogar über einen sicheren VPNTunnel außerhalb der Klinik mit dem KIS verbinden.

\section{APP für alle Mobilgeräte}

Der Hersteller Agfa Healthcare hat eine ähnliche APP entwickelt, die den mobilen Zugriff auf das von Agfa stammende KISSystem „Orbis” erlaubt. Die APP „Orbis ME" ist im Gegensatz zu der Lösung der Telekom plattformunabhängig - man kann sie also nicht nur mit Apple-Geräten, sondern auch mit allen anderen Mobilgeräten nutzen. „Angesichts der zunehmenden Nachfrage nach WindowsTablets und der Tatsache, dass solche Oberflächen auch besser in die klinische IT-Struktur hineinpassen und Apple für viele im Krankenhaus doch ein Fremdkörper darstellt, ist das ein großer Vorteil", so Oliver Nauroth, Geschäftsleiter für klinische Arbeitsplatzsysteme von Agfa Healthcare. Auch auf Android-Betriebssystemen läuft die APP. „Sie bietet im Prinzip den kompletten Lesezugriff auf Informationen administrativer Natur über den Patienten: Laborwerte, kodierte Diagnosen, Prozeduren, Eingriffe, OPs, Medikationen, Bilder - die kompletten Inhalte der Krankengeschichte also", erklärt Nauroth. Einzelne Aufträge an die Leistungsstellen wie dem Labor oder der Radiologie können Ärzte ebenfalls stellen. „Außerdem bieten wir auf den mobilen Geräten einen kompletten Medikationsarbeitsplatz, inklusive der Informationen, die aus einem Arzneimitteltherapiesicherheitssystem kommen", sagt Nauroth.

\section{Auf die Nutzer zugeschnitten}

Der Anbieter Nexus verfolgt dagegen eine etwas andere Strategie. Statt eine einzige APP zu entwickeln, die sich an den im KIS definierten Rechten ihrer jeweiligen Nutzer orientiert und ihren Anwendern nur

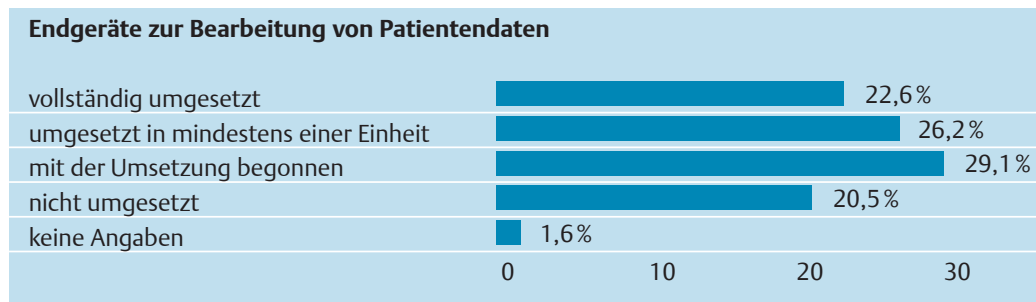

Abb. 3 Um ein mobiles endgerät nutzen zu können, brauchen Kliniken ein geschütztes drahtloses Netzwerk (WLAN). Vollständig umgesetzt ist das allerdings erst in rund $22 \%$ aller Krankenhäuser. Quelle: IT Report Gesundheitswesen 2013 den Zugriff auf jene Daten erlaubt, für die sie eine Berechtigung haben, bietet der Hersteller gleich mehrerer APPs an. Sie sind auf die einzelnen Teilprozesse in einer Klinik zugeschnitten und auf die jeweilige Aufgabe des Nutzers abgestimmt. „Wir richten die Strategie unserer mobilen Komponenten so aus, dass wir mit unseren APPs gezielt jene Informationen aus dem KIS herauslösen, die zur Bewältigung von Aufgaben in einem bestimmten Arbeitskontext benötigt werden", erklärt Oliver Schmid, der deutsche Vertriebsleiter von Nexus. So liefert Nexus zum Beispiel eine APP für die Notaufnahme oder die Geburtshilfestation. „Dafür haben wir einen speziellen APP-Store entwickelt, der dem Haus selbst die Wahlfreiheit gibt, bestimmte Prozesse zu mobilisieren und kontrolliert bereitstellen zu können", so Schmid. Um die Kontrolle über $\mathrm{Zu}$ griffsrechte zu behalten, stellt Nexus diese Auswahlmöglichkeiten nur den verantwortlichen EDV-Leitern zur Verfügung. Wie bei den anderen Anbietern funktionieren sowohl die einzelnen APPs als auch der APP-Store ausschließlich mit dem KIS von Nexus. Die APPs selbst kann man ebenfalls mit allen Mobilgeräten benutzen. Der Hersteller gibt den Kliniken mit dem Entwicklertool „APP Builder” außerdem die Möglichkeit, auf Basis der bestehenden APP-Entwicklungen individuelle Erweiterungen vorzunehmen.

Die Vorteile des mobilen KIS liegen auf der Hand: Wenn Ärzte und Pflegekräfte schnell, jederzeit und überall im Krankenhaus auf aktuelle Untersuchungsergebnisse ihrer Patienten zugreifen, können sie Fälle schneller abarbeiten und müssen nicht erst in der entsprechenden Abteilung nachfragen, ob etwa die Untersuchung schon gemacht wurde oder wann OP-Termine anstehen. Dass sie auch den Patienten zugute kommen, belegt eine Pilotstudie, die die Berliner Charité zusammen mit der Telekom durchgeführt hat. Demnach verkürzt sich nicht nur die Vor- und Nachbereitungszeit der Visite durch den Einsatz von Tablet-PCs, sondern verlängert sich auch die Zeit, die Ärzte dabei am Bett des Patienten verbringen.
Der Beitrag erschien zuerst in kma - Das Gesundheitswirtschaftsmagazin 2015; 20: 4749. 